\title{
The Research on Single-Disc Rotor Nonlinear Vibration Model and Mechanism
}

\author{
Min $\mathrm{Wu}^{1,2}$, Jinfu Yang ${ }^{1 *}$ \\ ${ }^{1}$ Institute of Engineering Thermophysics, CAS, Beijing, 100190, China \\ ${ }^{2}$ Full Three Dimension Power Engineering Co. Ltd., Beijing, 100190, China \\ *yangjinfu@mail.etp.ac.cn
}

\begin{abstract}
Keywords: Nonlinear vibration model, Duffing equation, Single-disc rotor, Nonlinear frequency, Frequency response equation, Research method
\end{abstract}

\begin{abstract}
Based on mechanical properties of rotor nonlinear vibration and the assumption of large deformation, the rotor nonlinear vibration model in the form of Duffing equation is constructed from typical engineering problems. According to analytical solutions of Duffing equation, system's equivalent frequency and frequency response equation are derived. Furthermore, the influences of stiffness, damping and nonlinear components are studied. The single-disc rotor nonlinear vibration experimental results verify the rationality and efficiency of nonlinear vibration model's construction and Duffing equation's analytical solution and frequency response equation. The mechanisms of resonance, amplitude jump and hysteresis are also revealed. It provides a new research method for rotor vibration characteristics analysis.
\end{abstract}

\section{Introduction}

Shaft rotor dynamics, which bases on shaft system movements which come from rotating machinery engineering, is a discipline that focuses on the relationships and laws between rotor dynamics and kinematics. The information and characteristics of vibration during operation are the main signals which can reflect the changing of the whole system. Through data acquisition (DAQ) and dynamic signal processing such as spectral analysis, coupled analysis [1-2] and wavelet analysis [3], we can gain the global and local rotor mechanism, to protect the normal operation and security of equipments, to prevent unexpected incidents.

At the aspect of nonlinear vibration model based on nonlinear components, T. Kapitaniak [4] considered the double-well potential Duffing oscillator model of nonlinear cubic stiffness in the periodic and random excitation, and discussed the reason of synchronization of bifurcation, chaos and other nonlinear vibration phenomenon in numerical results. Zang Chaoping et al [5-7] pointed out the limitations of linear models and considered the nonlinear factors of systems as determinants to recognize. They constructed a three-degree of freedom model contained nonlinear cubic stiffness, nonlinear square damping and uncertain factors of system parameters for constant displacement test and constant speed test to identify the nonlinear parameters. The results of maximum response prediction for the structures could be used to analyze the impact of nonlinear parameters for structural dynamics. At the aspect of applications in rotor systems, Li Hongkun et al [8] considered the loosening model of rotor-bearing systems contained the nonlinear cubic stiffness and nonlinear oil film force, and numerical results showed the feasibility of modeling method in rotor loosening fault diagnosis. Zhang Suohuai et al [9] derived the nonlinear dynamic model of gear coupled rotor-bearing systems according to nonlinear gear mesh stiffness, nonlinear bearing oil film force and gear backlash. Yuan Zhenwei et al [10] considered the nonlinear fluid exciting force model which could both reflect the radial eccentricity and axial deflection of turbine. Compared with the 
Alford force model, the numerical results showed the effectiveness and reliability of nonlinear fluid exciting force model. Ma Rui et al [11] comprehensively analyzed the gear crack evolution and the impact for bifurcation response of system dynamics through Poincare map and spectrum under the condition of nonlinear time-varying stiffness, nonlinear gear backlash and transmission errors, and carried out the crack fault diagnosis of gearbox. Li Ming et al [12] established the multi-span nonlinear rotor dynamic model contained nonlinear oil film force, and studied the bifurcation, chaos and other nonlinear dynamics of systems in numerical method. Besides, Guo Hulun et al [13] used analytical method to analyze two-degree of freedom airfoil model contained nonlinear cubic stiffness and nonlinear clearance, and gained the different bifurcations at flutter points under hard spring and soft spring characteristics. Wang Li et al [14] established the nonlinear model of flux linkage, and verified the validity of the nonlinear model.

Therefore, based on the assumption of large deformation, this paper uses nonlinear support stiffness described as hard spring characteristics to construct the nonlinear vibration model of rotor systems, and carries out corresponding experimental studies.

\section{Rotor nonlinear vibration model}

Jeffcott rotor models are traditionally based on the assumptions of small disturbances and small deformations, and elastic deformations satisfy the linear Hooke's law. However, when modern rotating machinery gradually develop to high-speed, light-duty and large flexible structures, rotor flexible departure is beyond the scope of small disturbances and has some nonlinear vibration characteristics in common. Therefore, there is an urgent need to establish the theoretical models combined with engineering problems for large disturbances and large eccentricity (displacement) conditions, which further reveal the mechanism of rotor nonlinear vibrations.
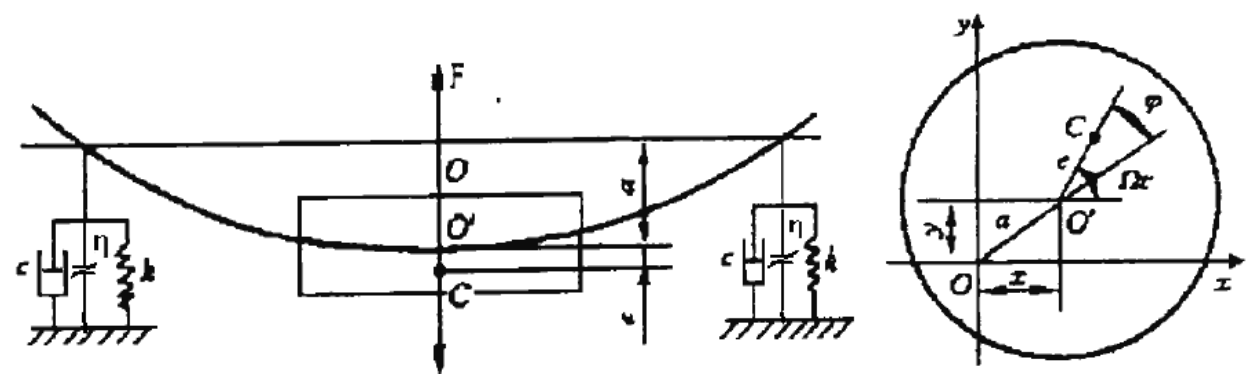

Fig. 1. Rotor force analysis

In Fig.1, the mass of the shaft is ignored; the shaft and bearings at both sides are elastic supports with large deformation and hard spring characteristics. Assume that the disc mass is $m$, eccentricity is $e$, rotational frequency is $\Omega$, with counter-clockwise positive, the inertial force components in $x, y$ axises are $m \ddot{x}_{C}=m(x+e \cos \Omega t)^{\prime \prime}$, $m \ddot{y}_{C}=m(y+e \sin \Omega t)^{\prime \prime}$, the damping force components in $x, y$ axises are $c \dot{x}$, $c \dot{y}$, the elastic restoring force components in $x, y$ axises are $k x+\eta x^{3}, k y+\eta y^{3}$. According to Newton's second law, the dynamic equations in $x, y$ axises as follows:

$$
m \ddot{x}+c \dot{x}+k x+\eta x^{3}=m \Omega^{2} e \cos \Omega t, \quad m \ddot{y}+c \dot{y}+k y+\eta y^{3}=m \Omega^{2} e \sin \Omega t
$$

The equation shows that rotor nonlinear vibration equations can be simplified into Duffing equation form of hard spring and damping. With $c / m=2 \mu \quad k / m=\omega_{0}^{2} \quad \eta / m=\varepsilon \beta_{0}^{2}(\varepsilon \rightarrow 0)$, Eq. (1) can be written as: 


$$
\begin{aligned}
& \ddot{x}+2 \mu \dot{x}+\omega_{0}^{2} x+\varepsilon \beta_{0}^{2} x^{3}=\Omega^{2} e \cos \Omega t \\
& \ddot{y}+2 \mu \dot{y}+\omega_{0}^{2} y+\varepsilon \beta_{0}^{2} y^{3}=\Omega^{2} e \sin \Omega t
\end{aligned}
$$

where $\Omega^{2} e$ indicates the amplitude of exciting force, is related to rotational frequency $\Omega$ and eccentricity $e . \mu$ indicates damping ratio. $\omega_{0}$ indicates natural frequency. $\varepsilon$ indicates small parameter coefficient. As the rotor nonlinear vibration equations are similar in either direction, so we discussed equations in $x$ axis in the following paper.

\section{Analytical analysis}

Without energy consumption when there is no damping component, Eq. (2) means conservative systems. When exciting force is ignored, the rotor nonlinear vibration of Duffing equation can be written as:

$$
\ddot{x}+\omega_{0}^{2} x+\varepsilon \beta_{0}^{2} x^{3}=0
$$

Using the method of multiple scales to derive the solutions [16] of Eq. (4). As the minimum level of approximation, substituted $\eta / m=\varepsilon \beta_{0}^{2}$, the system's equivalent frequency is $\omega=\omega_{0}+\frac{3 a^{2}}{8 m \omega_{0}} \eta$.

\section{Nonlinear vibration experiment of single-disc rotor system}

The single-disc rotor test bed shown in Fig.6. The rotor installs in bearing support, and connects to motor through coupling. Related parameters of the structure are: disc radius $r_{0}=0.039 \mathrm{~m}$, disc mass $m=0.5 \mathrm{~kg}$, shaft radius $r=0.005 \mathrm{~m}$, the material of disc and shaft is $\varphi 235$ steel, densities $\rho=7.8 \times 10^{3} \mathrm{~kg} / \mathrm{m}^{3}$, elastic modulus $E=200 \mathrm{Gpa}$, Poisson's ratio 0.3 , rotor $\operatorname{span} l=0.4335 \mathrm{~m}$. The horizontal and vertical eddy current sensors installed near the right side of the bearing are used to collect vibration signals from $x$ and $y$ axis. The key phase eddy current sensor installed near coupling is used to collect rotating speed. The vibration test system is shown in Fig.7, and the sampling frequency is $5000 \mathrm{~Hz}$.

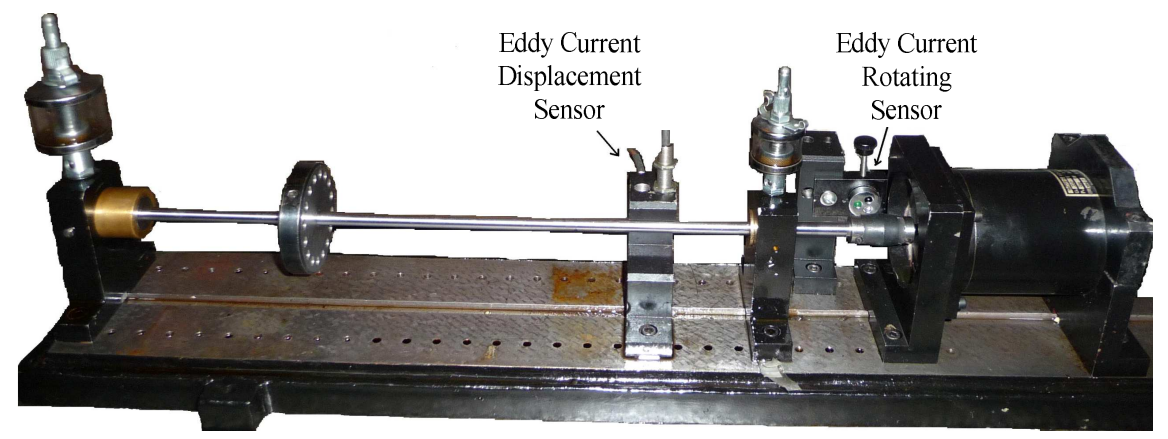

Fig. 6. Rotor test-bed 


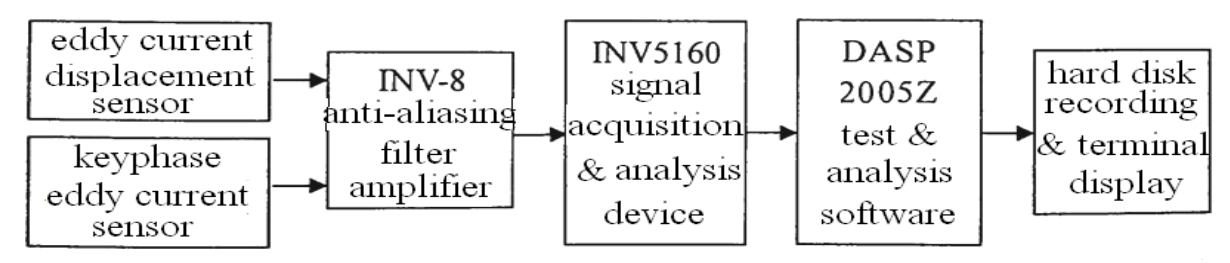

Fig. 7. Test equipment connection and test system

The disc which closes to the left side of the bearing has the distance $a=0.13005 \mathrm{~m}$. Rotor speed is directly controlled by adjustable speed motor, and the rotor acceleration and deceleration is tested respectively. In the rotor experiment, the rotor begins from 0rpm up to 9714rpm, and then down to 0rpm. The vibration responses of the critical speed in acceleration and deceleration are able to identify the resonance, whirl, and whip [24] and other typical fault features.

The structure frequency spectrum. The nonlinear vibration frequency spectrum characteristics during rotor acceleration and deceleration process is shown in Fig.8, where the horizontal axis means rotating frequency, vertical axis means running time, the depth of color means response amplitude value. The Fig.8 shows that in the start-stop process of rotor vibration test, there exists obvious simultaneous resonance region $\mathrm{A}$, oil whirl region $\mathrm{B}$ which changes frequency with operating speed, oil whip region $\mathrm{C}$ which doesn't change frequency with operating speed. In the acceleration process, rotor speed over the critical speed suddenly jump to a higher level, and from oil whirl to oil whip the whirl frequency suddenly jump to a lower level which called frequency locking phenomenon.

At $8030 \mathrm{rpm}$ to $9714 \mathrm{rpm}$ operating speed range, the oil whip phenomenon occurs, with corresponding whip frequency range from $62.59 \mathrm{~Hz}$ to $64.59 \mathrm{~Hz}$. The peak frequencies of the first bending critical speeds through acceleration and deceleration process are $73.91 \mathrm{~Hz}$ and $60.59 \mathrm{~Hz}$. At $7391 \mathrm{rpm}$ to $9714 \mathrm{rpm}$ range in acceleration process and $9714 \mathrm{rpm}$ to $8470 \mathrm{rpm}$ range in deceleration process, the oil whirl phenomenon occurs, with corresponding whirl frequency range from $61.26 \mathrm{~Hz}$ to $66.58 \mathrm{~Hz}$ and $62.59 \mathrm{~Hz}$ to $60.59 \mathrm{~Hz}$.

Nonlinear vibration analysis. Fig. 9 shows the amplitude-frequency curves through the critical speed during acceleration and deceleration process. Apparently, according to frequency response equation (13) and Fig.5(a), the experimental rotor vibration satisfies the hard spring properties that nonlinear coefficient $\eta$ greater than zero $(\eta>0)$, resulting rotor amplitude jump and hysteresis phenomena where amplitude suddenly falling at 4459rpm during acceleration process and suddenly rising at $4268 \mathrm{rpm}$ during deceleration process. 


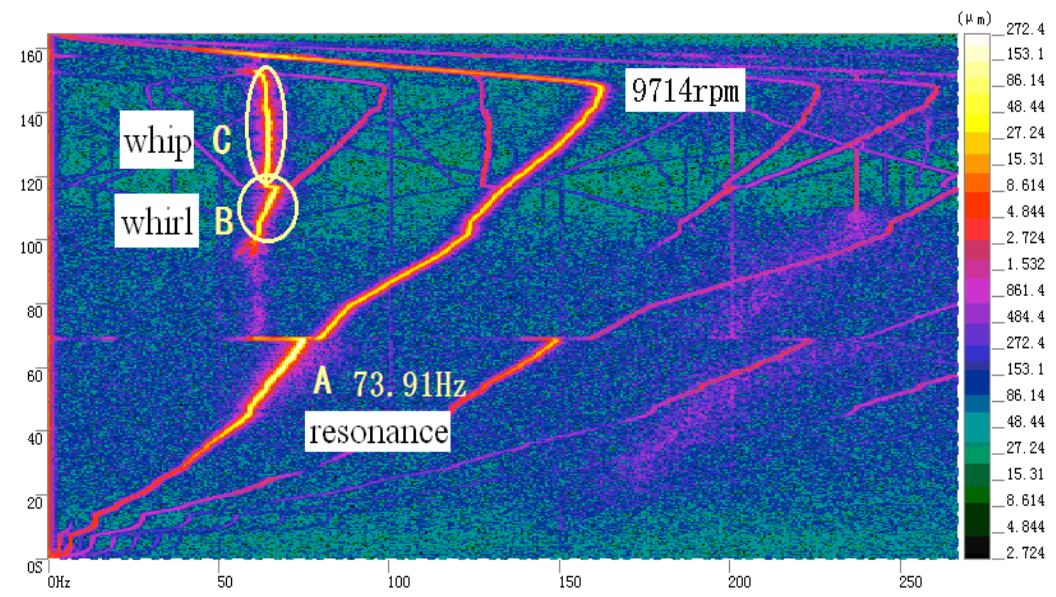

Fig. 8. Time-amplitude-frequency spectrum of rotor lifting process

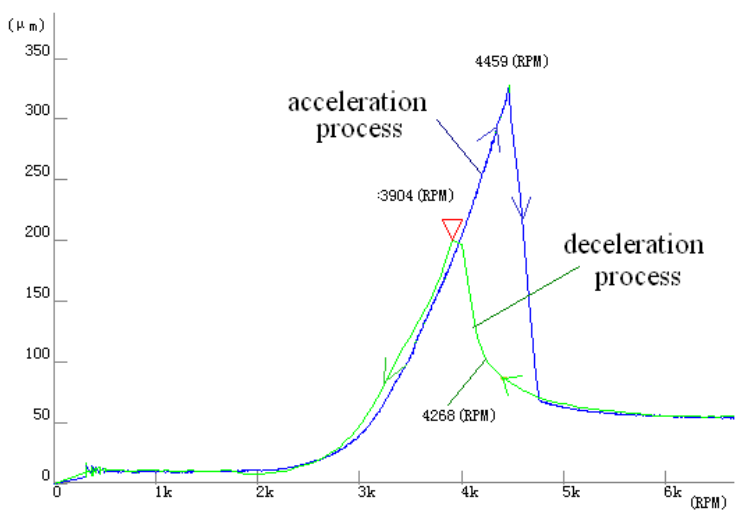

Fig. 9. Amplitude jump and hysteresis characteristic of rotor vibration

Fig.10 shows the shaft orbits and amplitude-frequency curve of rotor vibration through critical speed, bifurcation and chaos during acceleration process.

Fig.10 shows the shaft orbits in typical operating speed $(3570 \mathrm{rpm}, 4458 \mathrm{rpm}, 7375 \mathrm{rpm}$, 9583rpm) corresponding simultaneous vibration, simultaneous resonance, $1 / 2$ subharmonic bifurcation and $1 / 5$ subharmonic bifurcation, are respectively period-1 (<7000rpm), period-2 (7000 to $8000 \mathrm{rpm})$, period-5 (>8000rpm) of the motion features. Obviously, when rotor whirl frequency closes to the first natural frequency, $1 / 5$ subharmonic oscillation has two frequencies and low-frequency amplitude is higher than working frequency amplitude which called frequency locking.

\section{Conclusion}

In this paper, we use the basic principles of nonlinear vibration mechanics to construct the rotor nonlinear vibration model in the form of Duffing equation, and to derive the nonlinear system's equivalent frequency and frequency response equation, to study the mechanisms of resonance, amplitude jump, hysteresis and other nonlinear phenomena. Combined with single-disc nonlinear vibration experiment, we carry out in-depth study to reveal the nonlinear vibration mechanisms in acceleration and deceleration process, and verify the accuracy and reasonability of the nonlinear vibration modeling method, analytical solutions and frequency response equation. 


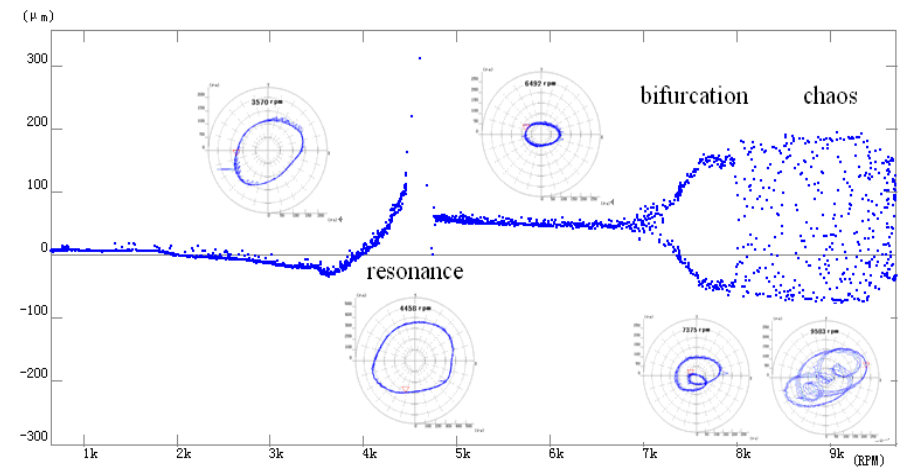

Fig. 10. Shaft orbits and amplitude-frequency curve of rotor vibration through critical speed, bifurcation and chaos

\section{Reference}

[1] J. Yang, K. Yang, D. Yu. Characteristics of nonlinear dynamic oil film forces and the decomposition of $\pi$ oil film forces [J]. Journal of Power Engineering, 2005, 25(6): 770-779.

[2] J. Yang, C. Chen, P. Yuan, W. Wu, C. Dong. Research on nonlinear fluid-solid interaction dynamic lubrication in floating ring bearings[J]. Journal of Aerospace Power [J], 2009, 24(2): 365-370.

[3] Z. Zhang, Y. Wang, W. Wang, J. Gu. Characteristics of rub-impact signal in phase space [J]. Journal of Xi' an Jiaotong University, 2001, 35(10): 1042-1049.

[4] K. Czolczynski, T. Kapitaniak, P. Perlikowski, A. Stefanski. Periodization of Duffing oscillators suspended on elastic structure: Mechanical explanation[J]. Chaos Solitons and Fractals. 2007, 32: $920-926$.

[5] G. Zhang, C. Zang. A novel method for nonlinear parameter identification based on vibration tests[C]. The $13^{\text {th }}$ national conference on nonlinear vibration, 2011, 078-08: 1-6.

[6] G. Zhang, C. Zang. Several issues on nonlinear parameter identification based on vibration tests[C]. The $13^{\text {th }}$ national conference on nonlinear vibration, 2011, 079-08: 1-9.

[7] G. Zhang, C. Zang. The influence of nonlinearities and uncertainties on the structural dynamics analysis[C]. The $13^{\text {th }}$ national conference on nonlinear vibration, 2011, 080-08: 1-11.

[8] H. Li, L. Zhao, Z. Zhang, Z. Guo. Characteristics analysis of nonlinear rigid rotor-bearing system with pedestal looseness fault $[\mathrm{C}]$. The $13^{\text {th }}$ national conference on nonlinear vibration, 2011, 044-11: 1-6.

[9] S. Zhang, S. Shi, D. Qiu. A nonlinear model for dynamic analysis of a geared rotor-bearing system[J]. Mechanical Science and Technology, 2001, 20(2): 191-193.

[10] Z. Yuan, F. Chu, R. Hao. A fluid-structure interaction model of turbine rotors based on a comprehensive leakage flow mechanism[J]. Engineering Mechanics, 2006, 23(12): 165-170.

[11] R. Ma, Y. Chen. Nonlinear mechanism research on spur gear pair with cracked failure[C]. The $13^{\text {th }}$ national conference on nonlinear vibration, 2011, 141-08: 1-9.

[12] M. Li, M. A. Nonlinear dynamics of flexible multi-rotor system supported on misaligned journal bearing[C]. The $13^{\text {th }}$ national conference on nonlinear vibration, 2011, 013-08: 1-9.

[13] H. Guo, Y. Chen. Analytical analysis of a 2-DOF Airfoil with freeplay and cubic Nonlinearity[C]. The $13^{\text {th }}$ national conference on nonlinear vibration, 2011, 146-08: 1-8.

[14] L. Wang, X. Song, X. Meng, Y. Yan. Nonlinear modeling of fielding-winding doubly salient generator by support vector machine [J]. Transactions of Electrotechnical Society, 2004, 19(9): $1-5$. 